\section{Commentary: Plasma exchange during extended normothermic ex vivo perfusion of donor hearts may lead to the Holy Grail of nonemergency transplantation!}

\author{
David J. Chambers, PhD
}

Extending the safe and viable preservation duration of donor hearts from the current limit of approximately 6 hours $^{1}$ to around 24 hours or longer has long been the ambition of surgeons and researchers in the field of cardiac transplantation studies. Dr Bartlett's group had previously demonstrated $^{2}$ that ex vivo isolated sheep hearts could be perfused for up to 72 hours (with good viability and function) when additional plasma cross-circulation exchange via a live awake paracorporeal sheep was used. In contrast, control nonplasma exchange hearts failed within 6 to 10 hours. It was appreciated, however, that this paracorporeal animal model would not be clinically realistic. Hence, in the present, proof-of-concept, study, ${ }^{3}$ this group explored the feasibility of maintaining ex vivo donor piglet hearts by continuous 24-hour blood perfusion and plasma exchange without the use of a paracorporeal animal. Plasma exchange involves the controlled addition of plasma (extracted from pooled porcine blood and infused at a rate of $1 \mathrm{~mL} / \mathrm{h} / \mathrm{kg}$ ) to an isolated heart perfusion circuit filled with donor blood and from which plasma was removed at the same rate, using a commercially available plasma separator. Control hearts did not receive the plasma exchange. Despite the small number of hearts used in this study (5 hearts/group), the outcome was striking and may represent

\footnotetext{
From Cardiac Surgery/Cardiac Surgical Research, The Rayne Institute (King's College London), Guy's and St Thomas' NHS Foundation Trust, St Thomas' Hospital, London, United Kingdom.

Disclosures: The author reported no conflicts of interest.

The Journal policy requires editors and reviewers to disclose conflicts of interest and to decline handling or reviewing manuscripts for which they may have a conflict of interest. The editors and reviewers of this article have no conflicts of interest.

Received for publication Dec 16, 2020; revisions received Dec 16, 2020; accepted for publication Dec 17, 2020; available ahead of print Dec 25, 2020.

Address for reprints: David J. Chambers, PhD, Cardiac Surgery/Cardiac Surgical Research, The Rayne Institute (King's College London), Guy's and St Thomas' NHS Foundation Trust, St Thomas' Hospital, 4th Floor, Lambeth Wing, London SE1 7EH, United Kingdom (E-mail: david.chambers@kcl.ac.uk).

J Thorac Cardiovasc Surg 2022;164:139-40 0022-5223/ $\$ 36.00$

Crown Copyright $(\subset) 2020$ Published by Elsevier Inc. on behalf of The American Association for Thoracic Surgery

https://doi.org/10.1016/j.jtcvs.2020.12.078
}

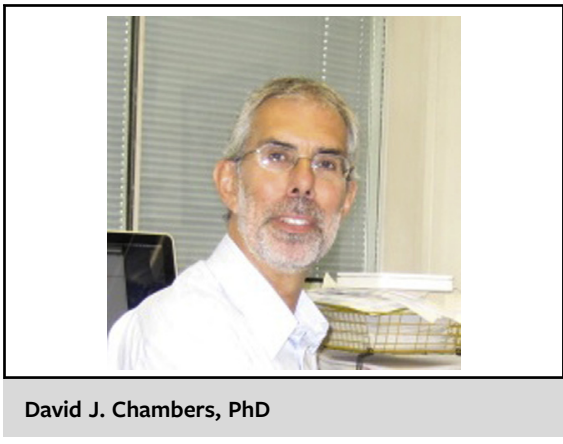

\section{CENTRAL MESSAGE \\ Continuous plasma exchange during 24-hour blood perfusion of ex vivo piglet hearts provides a potential clinically feasible outcome for heart transplantation.}

a significant leap forward for heart transplantation! All the plasma exchange hearts functioned well for 24 hours, with normal contractile and electrical activity, reduced lactate release, and improved oxygen use. However, 4 of the 5 control hearts had failed before 24 hours of perfusion.

The study poses a number of clinical challenges that would need to be addressed. It was conducted using piglet donor hearts, primarily because they are smaller and would require less volume of plasma during the exchange process. Since most heart transplantation involves adult patients, it would be important to use mature pig hearts in this system (immature hearts are known to be more tolerant of the hypoxic stress ${ }^{4}$ that this system may impose) and check the rate of plasma exchange that might be required. Would the authors envisage the use of fresh-frozen plasma as the infusion for any clinical plasma exchange scenario?

Another aspect that would benefit from further study relates to endothelial dysfunction during donor heart perfusion and storage. ${ }^{5}$ It is interesting that coronary resistance was greater, and pulmonary artery flow (equivalent to coronary flow) was lower, in the plasma exchange hearts compared with control hearts, when function was improved in the plasma exchange hearts. Perhaps an additive that helps maintain endothelial function, such as the novel nitric oxide-donor S-nitroso human serum albumin, ${ }^{6}$ may be potentially beneficial in the plasma exchange system to improve the endothelial function and maintain coronary flow. 
Also, it would be of interest (as has been indicated by the authors in their response to reviewers but not mentioned in the manuscript text) to conduct proteomic/metabolomic assessment of the infused plasma composition in comparison with the extracted plasma composition over the time of the perfusion; this might give more indication of the donor heart physiology during this perfusion period.

In conclusion, this pilot study represents a potentially very promising advance toward extending the viability of donor hearts for transplantation but, like most studies, leads to many more avenues that need exploring before it can be incorporated into the clinical armamentarium.

\section{References}

1. Hicks M, Hing A, Gao L, Ryan J, Macdonald PS. Organ preservation. Methods Mol Biol. 2006:333:331-74.

2. McLeod JS, Poling C, Church JT, Jung J, Sarosi E, Langley M, et al. Ex vivo heart perfusion for 72 hours using plasma cross circulation. ASAIO J. 2020;66:753-9.

3. Tchouta L, Drake D, Hoenerhoff M, Rojas-Pena A, Haft J, Owens G, et al. Twentyfour-hour normothermic perfusion of isolated ex vivo hearts using plasma exchange. J Thorac Cardiovasc Surg. 2022;164:128-38.

4. Ostadal B, Ostadalova I, Kolar F, Sedmera D. Developmental determinants of cardiac sensitivity to hypoxia. Can J Physiol Pharmacol. 2014;92:566-74.

5. Kevelaitis E, Nyborg NC, Menasche P. Coronary endothelial dysfunction of isolated hearts subjected to prolonged cold storage: patterns and contributing factors. J Heart Lung Transplant. 1999;18:239-47.

6. Semsroth S, Fellner B, Trescher K, Bernecker OY, Kalinowski L, Gasser H, et al. S-nitroso human serum albumin attenuates ischemia/reperfusion injury after cardioplegic arrest in isolated rabbit hearts. J Heart Lung Transplant. 2005;24: 2226-34.
See Article page 128.

\section{Commentary: Getting better with every beat. Extending the clock with ex vivo perfusion for heart transplantation}

\author{
Nandan Kumar Mondal, MSc, MPhil, PhD, ${ }^{\mathrm{a}}$ and \\ Ravi Kiran Ghanta, MD ${ }^{\mathrm{b}}$
}

Donor heart supply remains the fundamental limiter to heart transplantation. Despite a growing need for organs, only 1 in 3 hearts from organ donors are used for transplantation. ${ }^{1}$ To reduce the growing gap between organ supply versus need, expanded donor selection criteria, such as increased donor age and even donation after cardiac death, have emerged. The acceptance of more "marginal" donors must be balanced by the need to ensure appropriate organ quality. Furthermore, the 6-hour cold ischemic time window

\footnotetext{
From the Divisions of ${ }^{\mathrm{a}}$ Cardiothoracic Transplantation and Circulatory Support and ${ }^{\mathrm{b}}$ Cardiothoracic Surgery, Michael E. DeBakey Department of Surgery, Baylor College of Medicine, Houston, Tex.

Disclosures: The authors reported no conflicts of interest.

The Journal policy requires editors and reviewers to disclose conflicts of interest and to decline handling or reviewing manuscripts for which they may have a conflict of interest. The editors and reviewers of this article have no conflicts of interest.

Received for publication Dec 13, 2020; revisions received Dec 13, 2020; accepted for publication Dec 14, 2020; available ahead of print Dec 25, 2020.

Address for reprints: Ravi Kiran Ghanta, MD, Division of Cardiothoracic Surgery, Michael E. DeBakey Department of Surgery, Baylor College of Medicine, Houston, TX 77030 (E-mail: ravi.ghanta@bcm.edu).

J Thorac Cardiovasc Surg 2022;164:140-1

0022-5223/ $\$ 36.00$

Copyright $₫ 2020$ Published by Elsevier Inc. on behalf of The American Association for Thoracic Surgery

https://doi.org/10.1016/j.jtcvs.2020.12.068
}

Check for updates

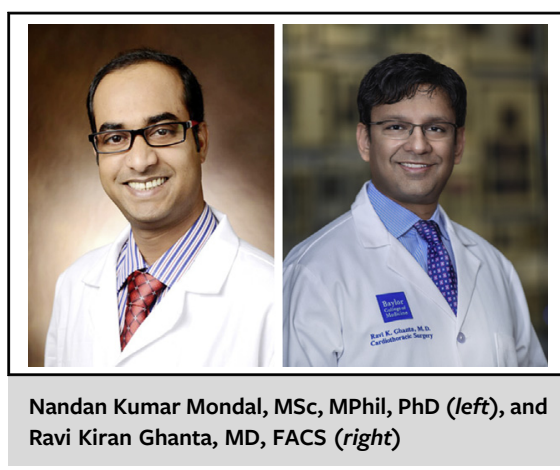

CENTRAL MESSAGE

Ex vivo heart perfusion is

possible using continuous

plasma exchange for a duration

of 24 hours, potentially expand-

ing time windows for evaluating

candidate organs for heart

transplantation.

remains a geographic and logistical hurdle for transplantation. Ex vivo organ perfusion systems have promise to overcome these challenges. The TransMedics Organ Care System has been used in heart transplantation and shown to be safe with normothermic perfusion times of less than 4 hours. $^{2}$

In this study, Tchouta and colleagues ${ }^{3}$ from the University of Michigan push the clock even further, demonstrating good explanted heart function 24 hours after explanation in pig hearts using normothermic ex vivo perfusion. This same 